\title{
EXPERIENCIA DEL MINISTERIO DE SALUD EN LA IMPLEMENTACIÓN DE LAS BRIGADAS DE MÉDICOS ESPECIALISTAS EN LAS ZONAS DE ASEGURAMIENTO UNIVERSAL EN PERÚ, 2009-2010
}

\begin{abstract}
Violeta Barzola-Cordero',a; Ena Llamosas²,b; Ciro Echegaray²,c; María Cuzco $2, a ;$ Felipe Peralta2,a
\section{RESUMEN}

Perú tiene alto grado de inequidad reflejado en la distribución de morbilidad-mortalidad, concordante con la inaccesibilidad a atención de salud y déficit de recursos humanos, especialmente en zonas alejadas de la capital. El Ministerio de Salud de Perú, reconociendo el derecho de toda la población a salud de calidad, inició el Aseguramiento Universal en Salud (AUS), en este marco, incrementó el acceso a atención especializada en zonas pilotos del AUS, mediante brigadas de médicos especialistas. Este artículo ofrece una aproximación a esta estrategia; presenta los procesos de gestión e implementación, hace un análisis cuantitativo con indicadores de producción/eficiencia, y brinda una mirada cualitativa desde la perspectiva de los brigadistas. Sus principales conclusiones inciden en la falta de gestión eficaz y eficiente, traducida en ausencia de: metas de producción, planes de trabajo, trabajo coparticipativo con actores locales, monitoreo y supervisión efectivos, reincidente escasez de recursos humanos y tecnológicos, y elevados costos.
\end{abstract}

Palabras clave: Estrategias Nacionales, Áreas de Especialidad, Desigualdades en la Salud, Recursos Humanos, Perú (fuente: DeCS BIREME).

\section{IMPLEMENTATION OF MEDICAL SPECIALISTS BRIGADES IN THE AREAS OF UNIVERSAL HEALTH COVERAGE: THE PERUVIAN MINISTRY OF HEALTH EXPERIENCE, 2009-2010}

\begin{abstract}
Peru has a high degree of inequity reflected in the distribution of morbidity and mortality, consistent with the inaccessibility to health care and human resource gap, especially in remote areas of the capital. The Peruvian Ministry of Health, recognizing the right of all people to quality health care, initiated the Universal Health Insurance (AUS), and in this context, increased access to specialized care in pilot AUS areas by brigades of specialist doctors. This article offers an approach to this strategy presents the management and implementation processes, a quantitative analysis with indicators of output / efficiency, and provides a qualitative look from the perspective of the members of the brigades. Its main findings reflect the lack of effective and efficient management, translated in the absence of: production goals, work plans, working partnerships with local stakeholders, monitoring and effective supervision, recurrent shortages of human and technological resources, and high costs.
\end{abstract}

Key words: National Strategies, Specializations, Health Inequalities, Human Resources, Peru (source: MeSH NLM).

\section{INTRODUCCIÓN}

Conociéndose la relación entre ingresos económicos y salud, y sus efectos bidireccionales ${ }^{(1,2)}$, en la escasez 0 ausencia de los primeros, es el Estado, a través de las políticas sociales, el llamado a garantizar el acceso de la población a servicios de salud de calidad.

Las graves desigualdades que existen en Perú, principalmente en materia económica y social, desmedran la salud de los más pobres, establecidos mayormente en las zonas rurales de la sierra y selva, situación evidenciada en el contraste que existe en los indicadores de morbimortalidad estimados por quintiles. Si bien a nivel macro, la información promedio por país, presenta mejoras graduales en lo relacionado a la mortalidad materna e infantil, se oculta que estos índices, en grupos de indígenas o afrodescendientes, no están disminuyendo, incrementándose entonces la desigualdad existente, reafirmando que Latinoamérica es la región con mayor desigualdad del planeta (3,4); por lo tanto la falta de acceso a servicios de salud de calidad, es un indicador cardinal de la exclusión social (5).

Por otro lado, en el contexto social, los seguros de salud cumplen una función importante, puesto que las mejoras en salud tienen un efecto substancial en el crecimiento económico y consiguiente desarrollo de la sociedad (6,7). Adicionalmente, en el aspecto de seguridad social se

\footnotetext{
Gerencia Central de EsSalud. Lima, Perú.

2 Dirección General de Gestión del Desarrollo de los Recursos Humanos, Ministerio de Salud. Lima, Perú.

a Médico; 'b Licenciado Obstetricia; ' ${ }^{\circ}$ Licenciado Economía.
} 
debe recordar que si el entorno económico se caracteriza por incertidumbre y volatilidad, se debe fortalecer simultáneamente la eficiencia y la solidaridad (8).

En este contexto, nació el Aseguramiento Universal en Salud en Perú (AUS) mediante Ley 29344 en abril del 2009. Política de Estado en materia de salud, que tiene como objetivo garantizar el derecho de las personas a acceder a la seguridad social de salud ${ }^{(9)}$, reconociendo que este acceso se efectuará en condiciones de calidad. Haciendo un paralelo con el modelo de Handler et al. ${ }^{(10)}$, donde el Sistema de Salud Pública es el elemento base para obtener resultados de calidad en salud, se espera que este proceso rinda frutos y beneficie a los peruanos, especialmente a los excluidos. Adicionalmente, se estableció que en el marco del AUS todas las instituciones aseguradoras tienen la obligación de financiar como mínimo el Plan Esencial de Aseguramiento en Salud (PEAS), en los tres niveles de atención, desde los aspectos preventivos hasta los recuperativos ${ }^{(11,12)}$.

En ese marco, el Estado debe garantizar la calidad de la atención de salud, y por ello, mejorar la calidad del desempeño de sus recursos humanos. Tarea complicada, por la disminuida proporción de estos en nuestro país, agudizada por el fenómeno de emigración brain drain o transferencia inversa de tecnología ${ }^{(13)}$. Solo entre 1990-2008, de Perú migraron 166 mil científicos e intelectuales, incluidos profesionales de salud ${ }^{(14)}$. Adicionalmente, el Estado debe asegurar la disponibilidad de recursos financieros, infraestructura y tecnología de salud para su óptimo desempeño.

El Ministerio de Salud de Perú (MINSA) estableció tres estrategias fundamentales: i) aseguramiento universal ii) descentralización y fortalecimiento del primer nivel de atención e iii) implementación de acciones específicas en las zonas donde las tasas de mortalidad materna e infantil son más altas ${ }^{(15)}$. Por ello, la Dirección General de Gestión del Desarrollo de Recursos Humanos del MINSA, implementó la Estrategia de Brigadas de Médicos Especialistas en las zonas pilotos del AUS. El presente artículo, brinda un panorama general de la experiencia de estas brigadas durante los años 2009 a 2010 y tiene como propósito sumar evidencia primaria en la iniciativa de construcción del sistema de Aseguramiento Universal en el Perú.

\section{GESTIÓN E IMPLEMENTACIÓN DE LAS BRIGADAS ESPECIALIZADAS AUS}

Reconociéndose que el número y la calidad de los trabajadores de salud son directamente proporcionales a la cobertura en inmunizaciones, alcance de la atención primaria y supervivencia de lactantes, niños y madres, es imperativo asegurar que el recurso humano, además de competente, se encuentre presente en número suficiente, donde y cuando se requiera atender las necesidades de salud de la población.

La Dirección General de Gestión del Desarrollo de Recursos Humanos del MINSA determinó que en las regiones más pobres del país, seleccionadas como zonas piloto AUS, existía un déficit de 552 especialistas para alcanzar el mínimo necesario que cumpla las horas establecidas, según la categoría del Establecimiento de Salud (EESS). Cuando profundizó el análisis, según la necesidad de especialistas por Unidades Productoras de Servicios (UPS), la brecha se incrementó a 1374 , en nueve departamentos y los distritos del Valle de los Ríos Apurímac y Ene (VRAE); con estas cifras se gestionó, ante el Ministerio de Economía y Finanzas, el financiamiento para implementar esta estrategia. Por otro lado, según el estudio de Prioridades de Médicos Especialistas ${ }^{(16)}$ del 2009, en Perú hay 25 especialidades prioritarias, 19 de las cuales fueron parte de las brigadas de médicos especialistas.

Esta estrategia comenzó en agosto de 2009, en EESS del I y II nivel de atención en las zonas pilotos del AUS, fueron 19 los EESS visitados en nueve departamentos de Perú: Ayacucho, Amazonas, Apurímac, Huancavelica, Junín, La Libertad, Lambayeque, Loreto y San Martín. Las brigadas estuvieron conformadas por 1-4 médicos; de 21 especialidades: Anatomía Patológica, Anestesiología, Cardiología, Cirugía General, Emergencia y Desastres, Endocrinología, Gastroenterología, Geriatría, Ginecología y Obstetricia, Medicina Familiar y Comunitaria, Medicina Interna, Neumología, Neurocirugía, Neurología, Oftalmología, Otorrinolaringología, Patología Clínica, Pediatría, Psiquiatría, Traumatología y Urología.

Participaron 52 especialistas, desde agosto de 2009 hasta noviembre de 2010 - marco temporal del presente análisis- realizándose 222 visitas en total ( 1 especialista = 1 visita). Los EESS destino fueron establecidos por el MINSA y las Direcciones Regionales de Salud (DIRESA). Pero se visitaron EESS aledaños no programados, por iniciativa individual de algunos médicos, no incluidos en este análisis. El traslado fue realizado por vía aérea, terrestre o fluvial, en varios traslados usaron dos medios de transporte.

Estos EESS fueron cabezas de Red, pero, la comunicación de los especialistas con el nivel central y otros EESS de la misma red fue escasa, se adujeron problemas de gestión y operatividad del equipo de comunicaciones, en algunas zonas no existe línea telefónica. De las 222 visitas, $58 \%$ se efectuaron en EESS del II nivel de atención, $37 \%$ en el I nivel y $5 \%$ en un Laboratorio referencial de DIRESA. Apurímac recibió la mayoría de visitas con $45,5 \%$ del total; Ayacucho $14 \%$; Huancavelica 12,6\%; San Martín 12,2\%; Amazonas 
Tabla 1. Costos de las actividades realizadas en función a los honorarios profesionales de los médicos especialistas, según establecimientos de salud asistidos por las brigadas especializadas AUS, en Nuevos Soles.

\begin{tabular}{|c|c|c|c|c|c|c|}
\hline \multirow[b]{2}{*}{ Departamento (EESS) } & \multirow[b]{2}{*}{ Especialidad } & \multirow{2}{*}{$\begin{array}{c}\text { Total } \\
\text { actividades }\end{array}$} & \multicolumn{4}{|c|}{ Estimación de costos } \\
\hline & & & $\begin{array}{l}\text { Sueldo } \\
15 \mathrm{~d}\end{array}$ & $\begin{array}{l}\text { Número de } \\
\text { salidas }\end{array}$ & $\begin{array}{c}\text { Costo } \\
\text { total }\end{array}$ & $\begin{array}{c}\text { Costo / } \\
\text { actividad }\end{array}$ \\
\hline \multirow{6}{*}{$\begin{array}{l}\text { Ayacucho } \\
\text { (H. R. Ayacucho) }\end{array}$} & Psiquiatría & 887 & 5300 & 7 & 37100 & 41,83 \\
\hline & Oftalmología & 685 & 5300 & 4 & 21200 & 30,95 \\
\hline & Medicina interna & 436 & 5300 & 2 & 10600 & 24,31 \\
\hline & Traumatología & 321 & 5300 & 2 & 10600 & 33,02 \\
\hline & Geriatría & 326 & 5300 & 2 & 10600 & 32,52 \\
\hline & Endocrinología & 342 & 5300 & 2 & 10600 & 30,99 \\
\hline \multirow{4}{*}{$\begin{array}{l}\text { Apurímac } \\
\text { (C.S.Z. Chincheros) }\end{array}$} & Cirugía general & 1303 & 5300 & 9 & 47700 & 36,61 \\
\hline & Medicina interna & 1793 & 5300 & 8 & 42400 & 23,65 \\
\hline & Ginecología y obstetricia & 533 & 5300 & 2 & 10600 & 19,89 \\
\hline & Pediatría & 1127 & 5300 & 8 & 42400 & 37,62 \\
\hline \multirow{8}{*}{$\begin{array}{l}\text { Huancavelica } \\
\text { (H.D. Huancavelica) }\end{array}$} & Anat.Patológica & 480 & 5300 & 5 & 26500 & 55,21 \\
\hline & Medicina interna & 282 & 5300 & 2 & 10600 & 37,59 \\
\hline & Oftalmología & 545 & 5300 & 7 & 37100 & 68,07 \\
\hline & Neurocirugía & 95 & 5300 & 2 & 10600 & 111,58 \\
\hline & Urología & 172 & 5300 & 5 & 26500 & 154,07 \\
\hline & Neumología & 138 & 5300 & 2 & 10600 & 76,81 \\
\hline & Patología clínica & $0^{*}$ & 5300 & 2 & 10600 & \\
\hline & Cardiología & 76 & 5300 & 2 & 10600 & 139,47 \\
\hline \multirow{7}{*}{$\begin{array}{l}\text { Apurímac } \\
\text { (Guillermo Díaz de la Vega) }\end{array}$} & Medicina interna & 631 & 5300 & 3 & 15900 & 25,20 \\
\hline & Cirugía general & 206 & 5300 & 2 & 10600 & 51,46 \\
\hline & Endocrinología & 1781 & 5300 & 10 & 53000 & 29,76 \\
\hline & Geriatría & 1075 & 5300 & 7 & 37100 & 34,51 \\
\hline & Anat.Patológica & 91 & 5300 & 1 & 5300 & 58,24 \\
\hline & Patología clínica & 1316 & 5300 & 9 & 47700 & 36,25 \\
\hline & Anestesiología & 68 & 5300 & 2 & 10600 & 155,88 \\
\hline \multirow{6}{*}{$\begin{array}{l}\text { Apurímac } \\
\text { (H. Hugo Pesce) }\end{array}$} & Traumatología & 1654 & 5300 & 11 & 58300 & 35,25 \\
\hline & Anat.Patológica & 682 & 5300 & 8 & 42400 & 62,17 \\
\hline & Cirugía general & 198 & 5300 & 2 & 10600 & 53,54 \\
\hline & Otorrinolaring. & 419 & 5300 & 2 & 10600 & 25,30 \\
\hline & Psiquiatría & 565 & 5300 & 6 & 31800 & 56,28 \\
\hline & Pediatría & 1868 & 5300 & 9 & 47700 & 25,54 \\
\hline \multirow{5}{*}{$\begin{array}{l}\text { Tarapoto } \\
\text { (H. de Apoyo II Tarapoto) }\end{array}$} & Anat.Patológica & 2262 & 5300 & 5 & 26500 & 11,72 \\
\hline & Oftalmología & 296 & 5300 & 3 & 15900 & 53,72 \\
\hline & Gastroenterología & 288 & 5300 & 3 & 15900 & 55,21 \\
\hline & Emergencia & 76 & 5300 & 1 & 5300 & 69,74 \\
\hline & Endocrinología & 359 & 5300 & 5 & 26500 & 73,82 \\
\hline \multirow{2}{*}{ Lambayeque (Mr Salas ) } & Ginecología y obstetricia & 590 & 5300 & 4 & 21200 & 35,93 \\
\hline & Pediatría & 382 & 5300 & 2 & 10600 & 27,75 \\
\hline San Martín (H. Moyobamba) & Patología clínica & 96 & 5300 & 1 & 5300 & 55,21 \\
\hline $\begin{array}{l}\text { Apurímac } \\
\text { (Lab. Diresa) }\end{array}$ & Anat.Patológica & 480 & 5300 & 1 & 5300 & 11,04 \\
\hline \multirow{4}{*}{$\begin{array}{l}\text { Amazonas } \\
\text { (H. Gustavo Lanata) }\end{array}$} & Medicina interna & 703 & 5300 & 3 & 15900 & 22,62 \\
\hline & Cirugía general & 511 & 5300 & 6 & 31800 & 62,23 \\
\hline & Ginecología y obstetricia & 966 & 5300 & 5 & 26500 & 27,43 \\
\hline & Pediatría & 254 & 5300 & 1 & 5300 & 20,87 \\
\hline $\begin{array}{l}\text { Amazonas } \\
\text { (H. Santa Maria de Nieva) }\end{array}$ & Medicina familiar y comunitaria & 781 & 5300 & 4 & 21200 & 27,14 \\
\hline Amazonas (C.S. Nieva) & Medicina familiar y comunitaria & 819 & 5300 & 4 & 21200 & 25,89 \\
\hline $\begin{array}{l}\text { San Martín } \\
\text { (C.S. Barranquita) }\end{array}$ & $\begin{array}{l}\text { Medicina familiar } \\
\text { y comunitaria }\end{array}$ & 469 & 5300 & 4 & 21200 & 45,20 \\
\hline San Martín (C.S. Huimbayoc) & Medicina familiar y comunitaria & 76 & 5300 & 2 & 10600 & 139,47 \\
\hline San Martín (C.S. Pongo de C.) & Medicina familiar y comunitaria & 272 & 5300 & 2 & 10600 & 38,97 \\
\hline $\begin{array}{l}\text { La Libertad } \\
\text { (H. Leoncio Prado) }\end{array}$ & Cirugía general & 289 & 5300 & 2 & 10600 & 36,68 \\
\hline \multirow{4}{*}{$\begin{array}{l}\text { Ayacucho } \\
\text { (H. de Campaña } \\
\text { por la Paz del Vrae) }\end{array}$} & Ginecología y obstetricia & 352 & 8000 & 2 & 16000 & 45,45 \\
\hline & Cirugía general & 170 & 8000 & 3 & 24000 & 141,18 \\
\hline & Anestesiología & 117 & 8000 & 3 & 24000 & 205,13 \\
\hline & Traumatología & 172 & 8000 & 2 & 16000 & 93,02 \\
\hline $\begin{array}{l}\text { Loreto } \\
\text { (C.S. San Lorenzo) }\end{array}$ & Medicina familiar y comunitaria & 572 & 5900 & 3 & 17700 & 30,94 \\
\hline $\begin{array}{l}\text { Junín } \\
\text { (H. San Martín de Pangoa) }\end{array}$ & Cirugía general & 245 & 5300 & 1 & 5300 & 21,63 \\
\hline Total & & 32470 & & 222 & 1205400 & 37,12 \\
\hline
\end{tabular}

* No fue posible determinar el total de actividades. 
10,4\%; Lambayeque seis visitas (2,7\%); Loreto tres visitas, La Libertad dos y Junín una (Tabla 1).

Cada profesional laboró 15 días/brigada, de lunes a sábado, ocho horas diarias, por la modalidad de locación de servicios. La retribución económica, según la lejanía de la zona, fue s/.5300 u s/.8000 nuevos soles (Tabla 1). Si bien el empleador era el nivel nacional, los niveles locales y regionales debían costear el alojamiento y alimentación.

EI PEAS, presenta un listado de condiciones, intervenciones y prestaciones en base al estudio de carga de enfermedad y lesiones, medidas en años de vida saludables perdidos e identifica 50 diagnósticos como causa del $72 \%$ de los años de vida saludables perdidos y los agrupa en 21 categorías ${ }^{(17,18)}$. Las brigadas brindaron atención en 17 de esas categorías: enfermedades neuropsiquiátricas, no intencionales, condiciones perinatales, enfermedades cardiovasculares, tumores malignos, enfermedades osteomusculares, deficiencias nutricionales, infecciones respiratorias, infecciosas y parasitarias, enfermedades del aparato respiratorio, enfermedades del aparato digestivo, diabetes, enfermedades genitourinarias, condiciones maternas, intencionales, enfermedades endocrinas y de la sangre, otros tumores y enfermedades de la piel.

\section{PRODUCCIÓN Y EFICIENCIA DE LAS BRIGADAS ESPECIALIZADAS AUS: ANÁLISIS CUANTITATIVO}

Se evaluó 222 informes, correspondientes a cada una de las visitas efectuadas por los brigadistas.

\section{EVALUACIÓN DE LA PRODUCCIÓN}

Se brindaron siete tipos de atención: consulta externa, atenciones por emergencia, interconsultas, capacitaciones y actividades de gestión, cirugías, procedimientos y visita hospitalaria; dependientes de la especialidad.

Tabla 2. Producción de las brigadas especializadas AUS de acuerdo a Establecimientos de Salud.

\begin{tabular}{|c|c|c|c|c|c|c|c|c|}
\hline Establecimiento de Salud & $\begin{array}{l}\text { Consulta } \\
\text { Externa }\end{array}$ & $\begin{array}{l}\text { Emer- } \\
\text { gencia }\end{array}$ & $\begin{array}{l}\text { Intercon- } \\
\text { sulta }\end{array}$ & Cap/Gest & Cirugía & $\begin{array}{c}\text { Procedi- } \\
\text { miento }\end{array}$ & $\begin{array}{c}\text { Visita } \\
\text { Hospitalaria } \\
\end{array}$ & Total \\
\hline $\begin{array}{l}\text { Hospital Reg. } \\
\text { Ayacucho }\end{array}$ & 2828 & 34 & 233 & 20 & 46 & 173 & 116 & 3450 \\
\hline $\begin{array}{l}\text { Centro de Salud } \\
\text { Zonal Chincheros }\end{array}$ & 2870 & 206 & 162 & 36 & 14 & 266 & 1202 & 4756 \\
\hline $\begin{array}{l}\text { Hospital D. } \\
\text { Huancavelica }\end{array}$ & 1037 & 63 & 80 & 26 & 12 & 528 & 175 & 1921 \\
\hline $\begin{array}{l}\text { Hospital Guillermo Diaz - } \\
\text { Abancay }\end{array}$ & 3032 & 43 & 129 & 59 & 68 & 1429 & 408 & 5168 \\
\hline $\begin{array}{l}\text { Hospital Hugo Pesce P. - } \\
\text { Andahuaylass }\end{array}$ & 2644 & 472 & 101 & 22 & 129 & 926 & 1214 & 5508 \\
\hline Hospital Tarapoto & 760 & 94 & 62 & 4 & 0 & 2347 & 84 & 3351 \\
\hline C.S. Salas & 645 & 81 & 0 & 1 & 0 & 233 & 12 & 972 \\
\hline $\begin{array}{l}\text { Hospital Apoyo I } \\
\text { Moyobamba }\end{array}$ & 0 & 0 & 0 & 0 & 0 & 96 & 0 & 96 \\
\hline Lab. Diresa Apurímac & 0 & 0 & 0 & 0 & 0 & 480 & 0 & 480 \\
\hline $\begin{array}{l}\text { Hospital Gustavo } \\
\text { Lanata Bagua }\end{array}$ & 1149 & 51 & 153 & 1 & 90 & 244 & 746 & 2434 \\
\hline Hospital Santa María de Nieva & 781 & 0 & 0 & 0 & 0 & 0 & 0 & 781 \\
\hline C.S. Nieva & 819 & 0 & 0 & 0 & 0 & 0 & 0 & 819 \\
\hline C.S. Barranquita & 398 & 8 & 0 & 51 & 0 & 0 & 12 & 469 \\
\hline C.S. Huimbayoc & 64 & 0 & 0 & 12 & 0 & 0 & 0 & 76 \\
\hline C.S. Pongo de Caynarachi & 268 & 0 & 0 & 4 & 0 & 0 & 0 & 272 \\
\hline Hospital Leoncio Prado & 273 & 0 & 0 & 0 & 10 & 4 & 2 & 289 \\
\hline $\begin{array}{l}\text { Hospital de Campaña } \\
\text { por la Paz del Vrae }\end{array}$ & 380 & 26 & 10 & 0 & 105 & 116 & 174 & 811 \\
\hline $\begin{array}{l}\text { C.S. San Lorenzo - } \\
\text { Datem del Marañón }\end{array}$ & 437 & 6 & 0 & 5 & 0 & 124 & 0 & 572 \\
\hline $\begin{array}{l}\text { Hospital San Martín } \\
\text { de Pangoa }\end{array}$ & 200 & 25 & 2 & & 5 & 6 & 7 & 245 \\
\hline Total & 18585 & 1109 & 932 & 241 & 479 & 6972 & 4152 & 32470 \\
\hline
\end{tabular}

*CAP/GEST: Actividades de capacitación y gestión. 
Para este estudio, consulta externa incluye actividades intramurales y extramurales. Capacitación y gestión incluye: difusión radial, elaboración de documentos de gestión, reuniones con las autoridades locales, charlas al personal en formación o profesionales locales. Procedimientos comprende: ecografías, cirugías menores, procedimientos de laboratorio, procedimientos efectuados en consulta externa y hospitalización, pericias psiquiátricas, terapias de grupo, biopsias, atención de parto normal e inmunizaciones.

Se brindaron 32470 atenciones, el 57\% fueron consultas externas. El Hospital Hugo Pesce de Andahuaylas tuvo la mayoría de atenciones, con 5508 (Tabla 2). Al evaluar la producción por especialidades, la que mayor número de atenciones efectuó fue Anatomía Patológica con 3 995, la mayoría fueron lecturas de láminas de citología exfoliativa. Los especialistas en Medicina Familiar y Comunitaria realizaron el mayor número de consultas externas, y la mayoría de visitas extramurales; no todas las especialidades efectuaron actividades extramurales (Tabla 3).

Siendo importante evaluar el grado de complejidad de los diagnósticos en consulta externa, por especialidad, se adoptó como parámetro de referencia la clasificación de capa simple aprobada por Decreto Supremo N. ${ }^{\circ}$
009-97-SA, para efectos de este análisis se denominan: diagnósticos de baja complejidad; los diagnósticos no registrados en este listado fueron considerados: diagnósticos complejos. Psiquiatría y Cardiología obtuvieron $100 \%$ de diagnósticos complejos (Tabla 4).

Hubo especialidades que establecieron alta proporción de diagnósticos complejos, pero no todos correspondían con la especialidad, por ejemplo Cirugía General, tuvo $79 \%$ de diagnósticos complejos, de 1571 consultas, pero la mayoría fueron diagnósticos médicos; en otra visita, brindó 200 consultas externas, pero solo el $2 \%$ de los diagnósticos fueron quirúrgicos.

Al analizar el grado de complejidad del diagnóstico por EESS, se halló que en el Hospital Guillermo Díaz de Abancay se registró la mayor proporción de diagnósticos complejos, seguido del Centro de Salud Huimbayoc en el Bajo Huallaga (Tabla 5).

\section{ESTIMACIÓN DE LA EFICIENCIA}

Los costos de las brigadas, para construir indicadores de eficiencia, se calcularon solo en base a los honorarios de los especialistas, no se contabilizó los gastos de transporte, alojamiento, alimentación, empleo

Tabla 3. Producción de las brigadas especializadas AUS de acuerdo a especialidad.

\begin{tabular}{|c|c|c|c|c|c|c|c|c|}
\hline Especialidad & $\begin{array}{l}\text { Consulta } \\
\text { externa }\end{array}$ & $\begin{array}{l}\text { Emer- } \\
\text { gencia }\end{array}$ & $\begin{array}{l}\text { Intercon- } \\
\text { sulta }\end{array}$ & Cap/gest & Cirugía & $\begin{array}{l}\text { Procedi- } \\
\text { miento }\end{array}$ & $\begin{array}{c}\text { Visita } \\
\text { hospitalaria }\end{array}$ & Total \\
\hline Anatomía Patológica & 0 & 0 & 0 & 15 & 0 & 3980 & 0 & 3995 \\
\hline Anestesiología & 0 & 4 & 0 & 1 & 104 & 0 & 76 & 185 \\
\hline Cardiología & 44 & 5 & 19 & 0 & 0 & 8 & 0 & 76 \\
\hline Cirugía General & 1571 & 263 & 117 & 7 & 107 & 224 & 633 & 2922 \\
\hline Emergencia y Desastres & 0 & 76 & 0 & 0 & 0 & 0 & 0 & 76 \\
\hline Endocrinología & 2234 & 19 & 169 & 10 & 0 & 19 & 31 & 2482 \\
\hline Gastroenterología & 210 & 9 & 42 & 5 & 0 & 10 & 134 & 410 \\
\hline Geriatría & 1161 & 0 & 49 & 50 & 0 & 2 & 139 & 1401 \\
\hline Ginecología y Obstetricia & 1121 & 103 & 6 & 3 & 89 & 652 & 467 & 2441 \\
\hline $\begin{array}{l}\text { Medicina Familiar y } \\
\text { Comunitaria }\end{array}$ & 2767 & 14 & 0 & 72 & 0 & 124 & 12 & 2989 \\
\hline Medicina Interna & 2576 & 124 & 222 & 18 & 0 & 21 & 884 & 3845 \\
\hline Neumología & 96 & 6 & 2 & 0 & 0 & 3 & 31 & 138 \\
\hline Neurocirugía & 52 & 22 & 15 & 3 & 0 & 3 & 0 & 95 \\
\hline Neurología & 453 & 6 & 26 & 5 & 0 & 2 & 94 & 586 \\
\hline Oftalmología & 1371 & 25 & 27 & 6 & 26 & 71 & 0 & 1526 \\
\hline Otorrinolaringología & 338 & 5 & 11 & 4 & 0 & 52 & 9 & 419 \\
\hline Patología Clínica & 0 & 0 & 0 & 0 & 0 & 1482 & 0 & 1482 \\
\hline Pediatría & 2465 & 146 & 38 & 26 & 0 & 34 & 922 & 3631 \\
\hline Psiquiatría & 996 & 55 & 149 & 13 & 0 & 118 & 121 & 1452 \\
\hline Traumatología & 986 & 226 & 31 & 3 & 145 & 157 & 599 & 2147 \\
\hline Urología & 144 & 1 & 9 & 0 & 8 & 10 & 0 & 172 \\
\hline Total & 18585 & 1109 & 932 & 241 & 479 & 6972 & 4152 & 32470 \\
\hline
\end{tabular}

*CAP/GEST: Actividades de capacitación y gestión. 
Tabla 4. Distribución de frecuencias de diagnósticos complejos según especialidad en las brigadas especializadas AUS.

\begin{tabular}{|c|c|c|c|}
\hline Especialidad & $\begin{array}{c}\text { Consultas } \\
\text { externas }\end{array}$ & $\begin{array}{c}\text { Diagnósticos } \\
\text { complejos }\end{array}$ & $\%$ \\
\hline Cardiología & 44 & 44 & 100 \\
\hline Cirugía General & 1571 & 1241 & 79 \\
\hline Endocrinología & 2234 & 2220 & 99 \\
\hline Gastroenterología & 210 & 133 & 63 \\
\hline Geriatría & 1161 & 1134 & 98 \\
\hline Ginecología y Obstetricia & 1121 & 871 & 78 \\
\hline Medicina Familiar y Comunitaria & 2767 & 1813 & 66 \\
\hline Medicina Interna & 2576 & 1983 & 77 \\
\hline Neumología & 96 & 66 & 69 \\
\hline Neurocirugía & 52 & 41 & 79 \\
\hline Neurología & 453 & 333 & 74 \\
\hline Oftalmología & 1371 & 1141 & 83 \\
\hline Otorrinolaringología & 338 & 153 & 45 \\
\hline Pediatría & 2465 & 1758 & 71 \\
\hline Psiquiatría & 996 & 996 & 100 \\
\hline Traumatología & 986 & 927 & 94 \\
\hline Urología & 144 & 131 & 91 \\
\hline Total & 18585 & 14985 & 81 \\
\hline
\end{tabular}

* Diagnósticos de baja complejidad: diagnósticos incluidos en el DS N. 009-97-SA.

† Diagnósticos complejos: diagnósticos no incluidos en el DS N. 009-97-SA.

Tabla 5. Grado de complejidad de diagnósticos de acuerdo a Establecimiento de Salud asistido en las Brigadas especializadas AUS.

\begin{tabular}{|c|c|c|c|}
\hline Establecimientos de Salud & $\begin{array}{c}\text { Consultas } \\
\text { externas }\end{array}$ & $\begin{array}{l}\text { Diagnósticos } \\
\text { complejos }\end{array}$ & $\%$ \\
\hline Hospital Regional Ayacucho & 2828 & 2433 & 86 \\
\hline Hospital de Campaña por la Paz del Vrae - Sivia & 380 & 333 & 88 \\
\hline Hospital Gustavo Lanata - Bagua & 1149 & 908 & 79 \\
\hline Hospital Santa María de Nieva - Condorcanqui & 781 & 400 & 51 \\
\hline C.S. Nieva & 819 & 463 & 57 \\
\hline Hospital Guillermo Diaz Abancay & 3032 & 3002 & 99 \\
\hline Hospital Andahuaylas Hugo Pesce P. & 2644 & 2056 & 78 \\
\hline Centro de Salud Zonal Chincheros & 2870 & 2238 & 78 \\
\hline Laboratorio Diresa & 0 & 0 & \\
\hline Hospital Departamental Huancavelica & 1037 & 900 & 87 \\
\hline Hospital San Martín de Pangoa & 200 & 84 & 42 \\
\hline C.S. Salas & 645 & 437 & 68 \\
\hline Hospital Leoncio Prado & 273 & 183 & 67 \\
\hline C.S. San Lorenzo - Datem Del Marañón & 437 & 351 & 80 \\
\hline Hospital Tarapoto & 760 & 598 & 79 \\
\hline Hospital Apoyo I Moyobamba & 0 & 0 & \\
\hline C.S. Barranquita & 398 & 338 & 85 \\
\hline C.S. Huimbayoc - C.S. Yarina & 64 & 58 & 91 \\
\hline C.S. Pongo & 268 & 203 & 76 \\
\hline Total & 18585 & 14985 & 81 \\
\hline
\end{tabular}

* Diagnósticos de baja complejidad: diagnósticos incluidos en el DS N. 009-97-SA.

† Diagnósticos complejos: diagnósticos no incluidos en el DS N. 009-97-SA. 
Tabla 6. Costo promedio por actividad realizada de acuerdo a especialidad en Nuevos Soles.

\begin{tabular}{lcccc}
\hline \multicolumn{1}{c}{ Especialidad } & Total actividades & N. ${ }^{\circ}$ salidas & Costo / Especialidad & Costo / Actividad \\
\hline Anatomía Patológica & 3995 & 20 & 106000 & 26,53 \\
Anestesiología & 185 & 5 & 34600 & 187,03 \\
Cardiología & 76 & 2 & 10600 & 139,47 \\
Cirugía General & 2922 & 25 & 140600 & 48,12 \\
Emergencia y Desastres & 76 & 1 & 5300 & 69,74 \\
Endocrinología & 2482 & 17 & 90100 & 36,30 \\
Gastroenterología & 410 & 4 & 21200 & 51,71 \\
Geriatría & 1401 & 9 & 47700 & 34,05 \\
Ginecología y Obstetricia & 2441 & 13 & 74300 & 30,44 \\
Medicina Familiar y Comunitaria & 2989 & 19 & 102500 & 34,29 \\
Medicina Interna & 3845 & 18 & 95400 & 24,81 \\
Neumología & 138 & 2 & 10600 & 76,81 \\
Neurocirugía & 95 & 2 & 10600 & 111,58 \\
Neurología & 586 & 3 & 15900 & 27,13 \\
Oftalmología & 1526 & 14 & 74200 & 48,62 \\
Otorrinolaringología & 419 & 2 & 10600 & 25,30 \\
Patología Clínica & 1482 & 13 & 68900 & 46,49 \\
Pediatría & 3631 & 20 & 106000 & 29,19 \\
Psiquiatría & 1452 & 13 & 68900 & 47,45 \\
Traumatología & 2147 & 15 & 84900 & 39,54 \\
Urología & 172 & 5 & 26500 & 154,07 \\
Total & 32470 & 222 & 1205400 & 37,12 \\
\hline
\end{tabular}

de recursos tecnológicos, medicamentos u honorarios de otros profesionales; por no disponerse de esa información, lo que es una limitante en el análisis; sin embargo, no deja de ser importante esta aproximación.

El costo total de la estrategia, en el periodo de estudio, para las 222 visitas, fue de un millón doscientos cinco mil cuatrocientos nuevos soles (S/.1205 400 nuevos soles). Considerando que se realizaron 32470 actividades, el costo promedio por actividad fue S/. 37,12. Las atenciones más costosas se brindaron en Sivia (VRAE) con un costo promedio de S/. 205,13; cantidad superior a los S/. 155,88 gastados para similares atenciones anestesiológicas en el Hospital Guillermo Díaz de la Vega. Por el contrario las atenciones más económicas se dieron en el Laboratorio Referencial de la DIRESA Apurímac, en Anatomía Patológica, con un gasto por prestación de S/.11,04 frente a los S/. 55,21 gastados para similares prestaciones, en Huancavelica (Tabla 1).

En costos por prestación por especialidad, Anestesiología, Urología y Cardiología, fueron las más caras. Las que demandaron menor costo por prestación fueron Medicina Interna, Otorrinolaringología y Anatomía Patológica (Tabla 6). Los costos hallados dependerían de la zona (demanda y accesibilidad locales), y de la complejidad de las prestaciones (tiempo de ejecución).

\section{PERCEPCIONES SOBRE LA ESTRATEGIA DE BRIGADAS AUS: ANÁLISIS CUALITATIVO}

Para este análisis se dispuso de dos fuentes, los informes escritos de los especialistas y la entrevista virtual realizada a éstos, se entrevistó a 21 de 30 médicos operativos entre el 30 de noviembre y el 16 de diciembre de 2010.

\section{ANALISIS DE FORTALEZAS, OPORTUNIDADES, DE- BILIDADES Y AMENAZAS}

Los brigadistas identificaron como principales fortalezas de la estrategia la mayor capacidad resolutiva, el trabajo en equipo y la motivación de los profesionales. Las oportunidades se enfocaron en el apoyo local existente y el marco legal favorable. Las debilidades se relacionaron con ausencia de plan de trabajo y metas claras, falta de recursos, remuneraciones bajas, incertidumbre sobre la continuidad de la estrategia, régimen laboral. Las amenazas se relacionaron con la escasa coordinación entre los tres niveles de gobierno, falta de apoyo local, escasos recursos locales, deficiente gestión local, barreras de accesibilidad cultural y geográfica, inexperiencia del MINSA en la implementación de la estrategia, escasa difusión del marco legal de AUS, fundamentalmente. 


\section{RECONOCIMIENTO DE DEFICIENCIAS}

El aspecto más resaltante, informado por escrito reiterativamente, fue la carencia de estructura para la atención en los EESS, específicamente escasez de recursos, tanto humanos como tecnologías de apoyo, hubo profesionales que operaron con equipo quirúrgico propio. En algunas zonas, por desconocimiento del marco legal, se negó la atención gratuita cuando correspondía.

En la entrevista virtual, la principal preocupación fue la carencia de formatos, equipamiento básico para procedimientos quirúrgicos mayores y menores, equipamiento básico de laboratorio, reactivos $\mathrm{y}$, al igual que lo reportado en los informes, faltó recurso humano, existieron zonas donde los cirujanos no realizaron intervenciones quirúrgicas sino consultas externas de enfermedades comunes que podrían ser abordadas por médicos generales, porque faltaron otros profesionales (Anestesiólogos y enfermeras entrenadas). Otra preocupación fue la falta de gestión del alojamiento y alimentación durante las brigadas.

La escasez de recursos es atribuida al Nivel Nacional del MINSA (22\% de los entrevistados), al Nivel Regional (39\%) y al Nivel Local (39\%).

En el último escenario, reconocen que en los EESS existen serias deficiencias en cuanto al conocimiento técnico del personal de salud $(11,25 \%)$, falta de motivación del personal $(11,24 \%)$, deficiencias en gestión $(10,22 \%)$ y deficiencias en la supervisión desde niveles superiores $(9,20 \%)$.

\section{CONVENIENCIA LABORAL DE LAS BRIGADAS}

Considerando que se trata de un servicio a terceros, que no incluye beneficios laborales, se exploró la percepción de los especialistas respecto al régimen laboral, enfocando tres aspectos: profesional, económico y social/ familiar; profesionalmente, el $90,5 \%$ cree que el régimen es conveniente; económicamente el $71,4 \%$ cree que es conveniente y desde la mirada socio/familiar solo el $33,3 \%$ considera que es conveniente.

Si bien estos resultados no concuerdan completamente con el análisis FODA - donde una debilidad importante es el régimen laboral de los brigadistas - disminuyen las opiniones favorables conforme se introduce la pregunta al ámbito personal.

\section{PROPUESTAS PARA MEJORAR}

La mayoría de entrevistados coincidió en que principalmente el aspecto remunerativo debe ser mejorado $(37 \%)$, incrementar insumos para las brigadas (32\%) e incrementar su tiempo de duración (18\%). Prioritariamente, no solicitan recursos para las brigadas, se observa más bien sus necesidades personales sobre las necesidades de la estrategia.

\section{REFLEXIÓN Y LECCIONES APRENDIDAS}

No se planificó ni buscó explícitamente como consigna de las brigadas, el compromiso con los actores locales, o el establecimiento de alianzas estratégicas en búsqueda de trabajo coparticipativo para solucionar problemas a ese nivel, los hechos informados corresponden a esfuerzos aislados. No se establecieron metas de producción por especialidades ni zonas designadas, ni se consensuó un plan de trabajo, de modo que se promoviera el trabajo en equipo para el cumplimiento de objetivos explícitos. Esto determinó que el trabajo efectuado fuera disperso, desordenado e irregular.

Existe ausencia de recursos tecnológicos y humanos en los EESS, las UPSS son incompletas o limitadas por el escaso recurso existente, esto no fue previsto en la implementación de la estrategia. Hubo ausencia de gestión que suministre recursos apropiados, ocasionándose subutilización de recurso humano capacitado, en desmedro de la calidad de la atención de la brigada. Faltó el establecimiento de monitoreo permanente y supervisión temporal -semestral o trimestral- que buscara medir el avance, capacitación y compromiso de los profesionales con la labor encargada, identificando en tiempo real los procesos vulnerables antes que éstos deterioren la estrategia implementada.

Los profesionales de las brigadas perciben inseguridad laboral, tanto por la modalidad de su contrato como por el entorno en el que se desempeñan, estos factores hacen peligrar la continuidad de los brigadistas. Existe desconocimiento de Normativa Sectorial, en Anestesiología los profesionales no brindaron atención pre-quirúrgica a los pacientes, limitándose a efectuar evaluación intra y post quirúrgica. Otros brigadistas desconocían el proceso de referencia-contrarreferencia.

Existe necesidad de estandarizar informes para determinar toda la producción. El formato debería solicitar datos puntuales: tamaño de la población asistida, número de horas programadas en cada actividad, tasas de morbilidad y mortalidad, entre otros, que permitan el cálculo de los indicadores necesarios que midan el avance y los logros de la estrategia. Existe necesidad de observar el marco legal a fin de no vulnerar la seguridad en la atención de salud, si un profesional no está capacitado ni facultado para ejercer determinada actividad no debe efectuarla. 
El contrato celebrado con los brigadistas no cumplió los parámetros de locación de servicios puesto que existió subordinación para el cumplimiento de un horario y asistencia a determinado lugar de forma exclusiva para ejecutar actividades dependientes.

\section{DISCUSIÓN}

Es indudable que Perú necesita mejorar el acceso de su población más necesitada a los servicios de salud y que estos deben ser de calidad, dado que los recursos disponibles son escasos ${ }^{(16)}$, se exige de las autoridades pertinentes un máximo desempeño en la gestión, de modo que se logre la mayor eficiencia posible.

El proceso de Aseguramiento Universal en Salud significa un gran paso en ese sentido, como principio fundamental de la reforma en salud, pero no hay que perder de vista que en cuestión de cobertura existe una brecha entre teoría y realidad ${ }^{(19)}$, brecha que se evidenció en la implementación de esta estrategia; es importante incrementar la participación local, a través de la descentralización y fortalecer el primer nivel de atención.

La gestión eficiente debe iniciarse en la planificación, estableciéndose objetivos claros, determinándose las actividades necesarias, tratando de involucrar al recurso humano desde el inicio, velando por el adecuado clima organizacional y efectuando las acciones que día a día se condigan con lo planificado. Los servicios de salud, incluyendo los brindados como estrategias de las políticas de salud, deben orientarse a satisfacer las necesidades de la población, entonces deberíamos preguntarnos ¿Cuál es el perfil de demanda en cada una de las zonas piloto del AUS?, ¿Qué recursos necesito para satisfacerla? ¿Dispongo de recurso humano suficiente? ¿Se dispone de recursos tecnológicos necesarios en estas zonas? -entendiéndose por recursos tecnológicos al conjunto de tecnologías, equipos, dispositivos médicos, medicamentos, insumos, sistemas organizacionales, ambiente, infraestructura, entre otros ${ }^{(20)}$.

Debería reevaluarse si son necesarias todas las especialidades en esta estrategia o si es mejor identificar la especialidad más eficaz o simplemente fortalecer las competencias de los médicos locales para cumplir los objetivos del primer nivel de atención. Una especialidad que ha demostrado cumplir con las expectativas de la población y de la estrategia, fue Medicina Familiar, ya que sus aportes también se evidenciaron en el ámbito administrativo, estos profesionales demostraron un desempeño con enfoque gerencial y no solo asistencial.

Respecto a los costos por actividad por especialidades, al comparar, solo las consultas externas, con lo que ofrece el Seguro Social (EsSalud) a sus especialistas, por cada consulta externa en horas extras, que oscila entre $\mathrm{S} / .10$ y S/.14, en zonas como Chincheros para dar un ejemplo, y en zonas menos alejadas lo ofrecido oscila entre S/. 8 y S/. 12; se evidencia que cualquiera de las consultas externas de esta brigada fueron superiores a las cifras mencionadas.

Queda entonces la tarea de no solo buscar las respuestas a las preguntas planteadas, sino que además está pendiente el efectuar un análisis sobre cómo incrementar la eficiencia de las estrategias que en el marco del AUS deban implementarse, para sobre ello planificar.

\section{Contribuciones de Autoría}

VBC participó en la concepción y diseño del trabajo, recolección/obtención de resultados, análisis e interpretación de datos, redacción del manuscrito, revisión critica del manuscrito y aprobación de su versión final. ELI, CE, MC y FP participaron de la concepción y diseño del trabajo, aprobación de su versión final, obtención de financiamiento y asesoría técnica o administrativa.

\section{Fuentes de Financiamiento}

Dirección General de Gestión del Desarrollo de los Recursos Humanos del Ministerio de Salud del Perú.

\section{Conflictos de Interés}

Los autores declaran no tener conflicto de interés en la publicación del presente artículo.

\section{REFERENCIAS BIBLIOGRÁFICAS}

1. Bonds $\mathbf{M H}$, Keenan DC, Rohani P, Sachs JD. Poverty trap formed by the ecology of infectious diseases. Proc Biol Sci. 2010; 277(1685):1185-92.

2. Zhang W, Bansback N, Anis AH. Measuring and valuing productivity loss due to poor health: A critical review.Measuring and valuing productivity loss due to poor health: $A$ critical review. Soc Sci Med. 2011; 72(2):185-92.

3. Organización Panamericana de la Salud (OPS). Capacidades en salud pública en América Latina y el Caribe: evaluación y fortalecimiento. Washington. DC: OPS; 2007.

4. Kliksberg B. La inequidad en el campo de la salud pública en América latina Una cuestión crucial. Rev Fac Cienc EconomLa inequidad en el campo de la salud pública en América latina Una cuestión crucial. Rev Fac Cienc Econom UNMSM. 1999;4(13):183-204.

5. Guerra de Macedo C. La extensión de la protección social en salud en el nuevo Estado latinoamericano, Caracas Venezuela, 2001. Rev CLAD Reforma y Democracia. 2001;19(1):1-12.

6. Seinfeld J, La Serna C. Avanzando hacia el aseguramiento universal: ¿cómo lograr la protección en salud de personas de ingresos medio bajos y bajos? Economía y Sociedad 64. Lima: CIES; 2007; 
7. Silva MT. Sistema único de salud: la experiencia brasileña en la universalización del acceso a la salud. Rev Peru Med Exp Salud Pública. 2009;26(2):251-7.

8. Titelman D, Uthoff A. El papel del aseguramiento en la protección social. Santiago de Chile: CEPAL; 2003.

9. Congreso de la República del Perú. Ley № 29344 Ley Marco del Aseguramiento Universal en Salud, del 9 de abril. El Peruano, 9 de abril de 2009, p. 394077-80.

10. Handler A, Issel M, Turnock B. A conceptual framework to measure performance of the public health system. Am J Public Health. 2001;91(8):1235-9.

11. Arce M. Implementación del aseguramiento universal en salud en regiones piloto del Perú. Rev Peru Med Exp Salud Publica. 2009;26(2):218-21.

12. Ministerio de Salud del Perú. Plan Esencial de Aseguramiento en Salud (PEAS). Plan de beneficio del PEAS. Lima: MINSA; 2009.

13. Mayta-Tristán P, Dulanto-Pizzorni A, Miranda JJ. Low wages and brain drain: an alert from Peru. Lancet. 2008;371(9624):1577.

14. Norza Pilar. Emigración de Recursos Humanos Calificados y sus Consecuencias Económicas y Sociales para el Desarrollo de ALC. Perspectiva de la Organización Internacional de la Migración para la Subregión Andina. Reunión Regional: La Emigración de Recursos Humanos Calificados desde Países de América Latina y el Caribe. Caracas, Venezuela 17 y 18 de Junio de 2009.
15. Ugarte O. Lineamientos de Gestión propuestos por el Ministro de Salud Óscar Ugarte Ubilluz. Lima: MINSA; 2009.

16. Zevallos L, Pastor R, Moscoso B. Oferta y demanda de médicos especialistas en los establecimientos de salud del Ministerio de Salud: brechas a nivel nacional, por regiones y tipo de especialidad. Rev Peru Med Exp Salud Publica. 2011;28(2):177-85.

17. Ministerio de Salud del Perú. Plan esencial de aseguramiento en salud (PEAS). Plan de beneficio del PEAS. Lima: MINSA; 2009.

18. Velásquez A. Carga de enfermedad en el Perú y las prioridades del plan esencial de aseguramiento universal. Rev Peru Med Exp Salud Publica. 2009; 26(2):22-31.

19. Madies C, Chiarvetti S, Chorny M. Aseguramiento y cobertura: dos temas críticos en las reformas del sector de la salud. Rev Panam Salud Publica. 2000;8(1-2):33-42.

20. Perú, Ministerio de Salud. Norma Técnica de Salud Categorías de establecimientos del Sector Salud. Lima: MINSA; 2010.

Correspondencia: Violeta Barzola Cordero.

Dirección: Jr. Los Licopodios 115, Urb. San Hilarión, Lima 36, Perú.

Teléfono: (511) 458-4945 / 99581-0447

Correo electrónico: vbarzola2@yahoo.es

\section{Consulte la versión electrónica de la Revista Peruana de Medicina Experimental y Salud Pública en}

\section{wWw.scopus.com}

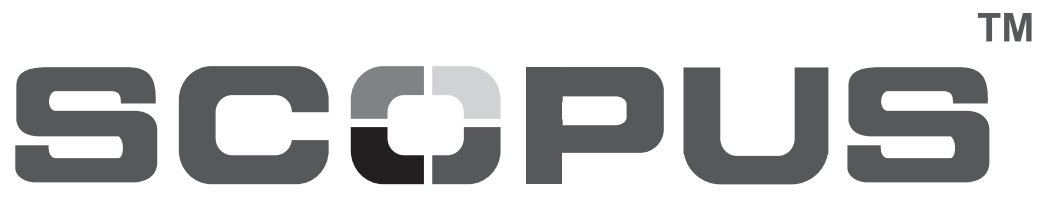

